\title{
Analysis of Common Plastic Chemical Phthalates to Fresh Water Fish (Tilapia Mossambica)
}

\author{
Dr. Y. Thangam ${ }^{1}$, S. Rajeswari ${ }^{2}$ \\ PG and research department of Zoology, J.K.K.Nataraja College of Arts and Science, Kumarapalayam, Mamakkal Dt, Tamilnadu, India
}

\begin{abstract}
Phthalates are the most commonly used plasticizers. It is one of the chemical used to make plastics. Phthalates in foods occur mainly as a result of contamination with phthalates from packaging materials. The intake of phthalates contained in food is the most significant source of exposure for humans. It has been established that the amount of phthalates found in foods or meals depends on the initial contamination of ingredients used in the production of the food, food production technologies, the period of storage (the time of contact with packaging materials), storage temperatures, ways of preparing dishes, the fat content in foods, and the type of packaging material used Phthalates are man-made chemicals used in many different products. Results obtained from this study show that percentage mortality of Tilapia mossambica fingerlings increased with increase in concentration of diethyl hexyl phthalate was dose dependent.
\end{abstract}

Keywords: Phthalates, Diethyl hexyl phthalate, Tilapia mossambica, bioassay studies

\section{Introduction}

Phthalates are often called plasticizers. Some phthalates are used as solvents (dissolving agents) for other materials. The phthalates widely selected to soften plastics are used because of their strong performance, durability and stability. These phthalates plasticizers are bound into the material in which they are added, they do not easily migrate out of the product or evaporate. Phthalates are the most commonly used plasticizers in the world and are categorized as high and low, depending on their molecular weight. Phthalates are used in hundreds of products in our homes, hospitals, cars and business. Phthalates (pronounced "thah-lates") are chemical plasticizers that have been widely used since the 1950 s to soften plastics. Colorless, odorless phthalates are not only cost effective, but also highly suitable for many flexible vinyl products. The characteristics of an individual phthalate often make it well suited to a particular product, allowing manufacturers to meet unique requirements for its use (function and safety specifications), appearance (texture, color, size and shape), and durability and wear. Phthalates are found in an amazing array of products. In personal care items, they're used to help lubricate other substances, help lotions penetrate and soften the skin, and help fragrances last longer. They're also used in toys, electronics (such as personal computers), car-care products, insecticides, and many household products, including adhesives, plastic wrap, plastic containers, flooring, furniture, wallpaper, shower curtains, and other things made of vinyl or PVC. Several of the chemicals used to produce plastics are hazardous for human health and the environment. These, and their degradation products, may be released during the life cycle of a plastic product. The overall aim of this paper is based on the study of the environmental and health hazards of plastics and the chemical phthalate DEHP (Diethyl hexyl phthalate). (Talsness et al. 2009).

Phthalates are detectable in aquatic environments, in dust and, because of their volatility, in air. There is considerable concern about the adverse effects of these chemicals on wildlife and humans. In addition to the reliance on finite resources for plastic production, and concerns about additive effects of different chemicals, current patterns of usage are generating global waste management problems. Barnes et al. (2009) show that plastic wastes, including packaging, electrical equipment and plastics from undo like vehicles, are major components of both household and industrial wastes. Phthalates, are organic substances often called Phthalic acid esters, frequently used in many industries. They are usually colourless or slightly yellowish oily and odourless liquids very slightly soluble in water. Phthalates are much more readily soluble in organic solvents, side chains are longer, and their lipo solubility and the boiling point are higher. Phthalates have a broad variety of uses. They are used as the so-called plasticisers that improve mechanical properties of plastic materials, mainly PVC. They are also used in the manufacture of floorings, children's toys, and are added to printing inks and to

perfumes and nail varnishes. Phthalates provide plasticity to rigid materials such as polyvinyl chloride and other polymers. They also lubricate, act as solvents, and impart favourable characteristics to products. Phthalates are exposed from blood bags, injection syringes, intravenous canellas and catheters, and from plastic parts of dialysers is also a possibility. Phthalate ingestion may occur via food, including enteric nutritional formulas, pharmaceuticals, nutritional supplements, sucking children's toys and other mouthing objects. Skin may come into direct contact with phthalate containing clothing, cosmetics, sunscreens, insecticides, other personal care products, modelling clay, toys, yoga pads, waxes, cleaning products and denture material. Many reports have discussed the impact of manmade xeno estrogenic compounds on man and wildlife. Phthalate is an industrial chemical that originated from a variety of compounds of anthropogenic origin such as pesticides, detergents and plasticizers used in products such insecticide, mosquito repellants, camphor substitute, plasticizer for cellulose, bathing soaps, cosmetics, pharmaceutical coal- tings, after shave-lotion, detergent, ester plastic film and sheets. (Huang et al., 2008).

Tilapia is a good biological model for toxicological and immunotoxicity studies (Perez et al., 2008) due to diverse characteristics, namely their high growth rates, efficiency in adapting to diverse diets, great resistance to diseases and handling practices, easy reproduction in captivity at prolific 


\section{International Journal of Science and Research (IJSR) \\ ISSN (Online): 2319-7064}

Index Copernicus Value (2015): 78.96 | Impact Factor (2015): 6.391

rate and finally, good tolerance to a wide range of environmental conditions. In human, phthalates have been detected in matrices such as blood, urine, saliva, amniotic fluid, breast milk and cord blood. The major pathway of exposure to phthalates is the oral route, though inhalation and dermal absorption may play a significant role in exposure (Adibi et al., 2003). Phthalates have been reported to affect multiple biochemical processes in humans and wildlife. These include effects on reproduction, damage to sperm (Rozati et al., 2002), early onset of puberty in females (Wolff, 2010), anomalies of reproductive tract (Desdoitslethimonier et al.,2012), infertility (Tranfo, 2012) and adverse outcomes pregnancy (Wang et al.,2009), to neuro developement (Miodovnik et al.,2011) and allergies (Bornehag et al.,2004). Because humans and wildlife can be exposed simultaneously to several phthalates any assessment of the risks posed by phthalates needs to consider combined effects of all of the phthalates in mixtures. To do this requires knowledge of the critical mechanisms of toxic action (CMTA) of each phthalate. Hence in the present investigation an attempt was made to study the impact of DEHP (Diethyl hexyl phthalate) to study the environmental and health hazards of chemicals in plastic polymers and products from a toxicological perspective. This was achieved by evaluations of toxicity by standardised toxicity tests and by identifying the hazards of chemicals Diethyl hexyl phthalate used to produce different plastic polymers.

The intake of DEHP phthalates contained in food is the most significant source of exposure for humans. It has been established that the amount of phthalates found in foods or meals depends on the initial contamination of ingredients used in the production of the food, food production technologies, the period of storage (the time of contact with packaging materials), storage temperatures, ways of preparing dishes, the fat content in foods, and the type of packaging material used. A factor which may significantly increase phthalate concentrations in animal tissues and, subsequently, in foods is their fat content. The lipophilic character of phthalates was also demonstrated by measurements of phthalate concentrations in water, milk and dairy products (Prokůpková et al. 2002). While the concentrations of DEHP in water samples varied from 0.49 $\mu \mathrm{g} / \mathrm{l}$ (deionised water) to $9.78 \mu \mathrm{g} / \mathrm{l}$ (mineral water in glass bottles with metal caps and PVC seals) (Prokůpková et al., 2002), reported total phthalate amounts between 0.06 and $0.32 \mathrm{mg} / \mathrm{kg}$ (of which DEHP $<0.01-0.09 \mathrm{mg} / \mathrm{kg}$ ) in pooled milk samples and $19.00 \mathrm{mg} / \mathrm{kg}$ in cream samples (DEHP $\max .2 .70 \mathrm{mg} / \mathrm{kg}$ ). The absolutely maximum concentration was found in cheese $(114 \mathrm{mg} / \mathrm{kg}$ total phthalates, $17 \mathrm{mg} / \mathrm{kg}$ DEHP).The fish Tilapia mossambica is the most common among Indian carps of Indo Ganges tic foot plains of Bangladesh, India and Pakistan. Specimens of Tilapia a fresh water teleost were selected for the investigation.

\section{Toxicant:}

DEHP (Diethyl hexyl phthalate) was used as a toxicant in the present investigation. Analytical grade of phthalate was obtained from (CAS no. 117-81-7, purity $>99 \%$ ) Loba chemise Pvt. Ltd., Mumbai, India.

\section{Structure of phthalate}<smiles>[R]OC(=O)c1ccccc1C(=O)O</smiles>

Diethyl hexylphthalate (DEHP)<smiles>CCCCC(CC)COCC(CC)(CC)COC(=O)c1ccccc1C(=O)O</smiles>

\section{Method}

Two types of methods were identified, they are the Continous flow method and Static method of the two types, the static method (APHA, 1998) were followed for the mortality study.

\section{Result}

Results obtained from this study show that percentage mortality of Tilapia mossambica fingerlings increased with increase in concentration of diethyl phthalate was dose dependent.

Table 1: Cumulative percentage mortality of Tilapia mossambica exposed to graded concentrations of diethyl phthalate (DEHP) for a maximum of $96 \mathrm{hrs}$

\begin{tabular}{|c|c|c|c|c|c|}
\hline DEHP & \multicolumn{5}{|c|}{ MORTALITY } \\
\hline$(\mu \mathrm{g} / \mathrm{L}) \%$ & $(24 \mathrm{~h}) \%$ & $(48 \mathrm{~h}) \%$ & $(72 \mathrm{~h}) \%$ & $(96 \mathrm{~h}) \%$ & Total \% \\
\hline Control & 0 & 0 & 0 & 0 & 0 \\
\hline 50 & 3.3 & 13.3 & 10 & 3.3 & 29.9 \\
\hline 75 & 6.7 & 16.7 & 13.3 & 10 & 46.7 \\
\hline 100 & 23.3 & 23.3 & 6.7 & 3.3 & 56.6 \\
\hline 150 & 43.3 & 26.7 & 20 & 10 & 100 \\
\hline
\end{tabular}

\section{Discussion}

Phthalates are a family of chemicals used in many consumer products, including building materials, household furnishings, clothing, cosmetics, personal care products, pharmaceuticals, nutritional supplements, herbal remedies, medical devices, denatures, children's toys, glow sticks, modelling clay, food packaging, automobiles, lubricants, waxes, cleaning materials and insecticides. Annually, more than three million metric tonnes of phthalates are produced globally (Bizzari et al., 2000). Uses of the various phthalates depend in part on their molecular weight (MW). Higher MW di(2-ethylhexyl) phthalate (DEHP are the phthalates produced in highest volume for use in construction material, clothing and furnishings. Phthalates used as a mixtures in single application. Because of their widespread use, all populations of people, domestic animals, and wildlife regularly encounter opportunities for exposure to phthalates. This paper reviews data regarding various sources and pathways of human exposure to phthalates from consumer products. Diethyl hexyl Phthalate (DEHP) is used as a 


\section{International Journal of Science and Research (IJSR) \\ ISSN (Online): 2319-7064}

Index Copernicus Value (2015): 78.96 | Impact Factor (2015): 6.391

plasticizer, a detergent base, in aerosol sprays, as a perfume binder and after shave lotion. It is known to be a contaminant of fresh water and marine ecosystem. Therefore, a study was designed to determine the acute toxicity effects of DEHP on a fresh water fish, Tilapia mossambica fingerlings. The fish was treated with 50, 75, 100 and $150 \mu \mathrm{g} / \mathrm{l}$. DEHP was dissolved in distilled water to determine the LC50. There was $100 \%$ mortality observed in $150 \mu \mathrm{g} / \mathrm{l}$. The LC50 of DEHP was estimated at log toxicant concentration for the total death. This shows that the impacts are dose and time dependent with respect to marked reduction in mortality rate. At sub-lethal concentrations of the test substance at 30, 40,60 and $80 \mu \mathrm{g} / 1$ in a renewal bioassay system, the water and the test compound were changed intermittently. One group was maintained as a control in dechlorinated water.

This study shows that percentage mortality of Tilapia mossambica fingerlings increased with increase in concentration of diethyl phthalate and was dose dependent. This is in consonance with a similar study by Nivedita et al., (2002) on toxic effect of DEHP on a fresh- water fish, Tilapia. The LC50 reported in this study is less than the observed field concentration in the water column $(0.16$ to $3.53 \mathrm{mg} / \mathrm{L}$ ) and sediment (0.16 to $0.32 \mathrm{mg} / \mathrm{L})$. Since DEHP binds to the sediment and remains in the water column, it is possible that it could pose serious threat to fish and other aquatic life. The rapid opercula movement, erratic swimming and loss of balance observed in this study. Results obtained from this study show that percentage mortality of Tilapia fingerlings increased with increase in concentration of diethyl phthalate and was dose dependent. Becker et al., (2004) reported levels of DEHP in the house dust of 254 children whose urinary metabolites of DEHP were also measured. The mean house dust level was $508 \mathrm{lg}$ $\mathrm{DEHP} / \mathrm{gm}$ dust, with no correlation between house dust levels and urinary levels of DEHP metabolites in the sample, suggesting that house dust is not a major contributor to total DEHP exposure. Another study of phthalate exposure via inhalation using personal air monitors also found no significant correlation between DEHP air levels and the urinary monoester metabolite, mono ethylhexyl phthalate (MEHP). Analysed phthalate levels in indoor air of 27 houses. They reported median concentrations of DEHP of $0.10,0.39,0.01,0.07$, and $0.11 \mathrm{lg} / \mathrm{m} 3$ respectively. For an adult breathing $20 \mathrm{~m} 3 /$ day, these would result in inhalation exposures of $2,78,0.2,1.4$ and $22 \mathrm{lg} /$ day respectively. Inhalation of contaminated dust will result in larger inhalation exposures.

\section{Conclusion}

Phthalate exposures in the general population and in subpopulations are ubiquitous and sources are widely variable. In the general population, the diet is generally considered the major pathway of exposure but all sources, path- ways, and their relative contributions to measured body burdens of phthalates are not well understood. Phthalates may be present but unidentified in many consumer products, including cosmetics, personal care products, home furnishings, pharmaceuticals, nutritional supplements and insecticides. In some instances, these may be important but unquantified sources of exposure. Oven baking of polymer clays may cause short-term, high-level inhalation exposures. Medical devices made of PVC containing DEHP are an important source of exposure to this reproductive and developmental toxicant in susceptible populations. Consumer products containing phthalates can result in human exposures through direct contact and use, by leaching into other products, or via general environmental contamination.

\section{References}

[1] TalsnessCE, AndradeAJ, KuriyamaSN, TaylorJA, VomSaalFS. Components of plastic: experimental studies in animals and relevance for human health. Trans. R. Soc. B 364, 2079-2096. 2009.

[2] BarnesDKA, GalganiF, ThompsonRC, BarlazM. Accumulation and fragmentation of plastic debris in global environments. Trans. R. Soc. B 364. 2009.

[3] HuangPC, KuoPL, GuoYL, LiaoPC, LeeCC. Associations between urinary phthalate monoesters and thyroid hormones in pregnant women. Hum. Reprod. 22, 2715-2722. 2008.

[4] PérezS, FarréMl, BarcelóD. Analysis, behavior and ecotoxicity of carbon-based nanomaterials in the aquatic environment. TrAC Trends Anal. Chem. 28, 820832.2008,

[5] AdibiJJ, PereraFP, JedrychowskiW, CamannDE, BarrD, JacekR, WhyattRM. Prenatal exposures to phthalates among women in New York City and Krakow, Poland. Environ. Health Perspect. 111, 1719-1722. 2003.

[6] RozatiR, ReddyPP, ReddannaP, MujtabaR. Role of environmental estrogens in the deterioration of male factor fertility. Fertil. Steril. 78, 1187-1194. 2002.

[7] WolfJ. Peroxisomes as dynamic organelles: peroxisomal matrix protein import. Hum Reprod. (16): 3268-78. 2010.

[8] Desdoits-LethimonierC,

AlbertO, Le

BizecB, PerduE, ZalkoD, CourantF,

LesnéL, GuilléF, Dejucq-RainsfordN, JégouB. Human testis steroidogenesis is inhibited by phthalates. FEBS J 277. 92: 77-88. 2012.

[9] TranfoG. Urinary phthalate monoesters concentration in couples with fertility problems. Tox Lett. 213 (1): 15-20. 2012.

[10] WangY, MathewsVA, KalnAJ. Short term exposure of neuroanatomical temperament and induce change in circuitry of adolscents. Brain imaging behaviour 3: 3850. 2009.

[11] MiodovnikKA, EngelSM, ZhuC, YeX, SooryLV, SilvaMJ. Environmental neurotoxicants and developing brain. J Med. 78(1): 58-77. 2011.

[12] BornehagCG, SundellJ, WeschlerCJ, SigsgaardT, LundgrenB, HasselgrenM, Hagerhed-EngmanL. The association between asthma and allergic symptoms in children and phthalates in house dust: a nested casecontrol study. Environ. Health Perspect. 112, 13931397. 2004.

[13] ProkupkovaG, HoladovaK, PoustkaJ, HajslovaJ. Development of a solid-phase microextraction metod for the determination of phthalic acid esters in water. Analytica Chimica Acta, 457: 211-223. 2002.

\section{Volume 6 Issue 1, January 2017




\section{International Journal of Science and Research (IJSR) \\ ISSN (Online): 2319-7064}

Index Copernicus Value (2015): 78.96 | Impact Factor (2015): 6.391

[14] APHA. Standard method for examination of water and water analysis. American public health association. 1998.

[15]BizzariN, JaeckelM, YoshidaY. Phthalates as a chemicals used in products, markering research report. SRI Int Pub. 24: 34-38. 2000.

[16] Niveditha, H., Thangavel,P., Dawood, A. K. S. and Ramaswami, M. 1998. Adaptive changes in the patterns of carbohydrate metabolites in blood, liver, Review of earlier work 44 muscle and heart tissues of Sarotherodon mossambicus (Peters) exposed to the carbamate fungicide ziram. Pestic. Sci., 52: 133-137.

[17] BeckerK, SeiwertM, AngererJ, HegerW, KochHM, Nag orkaR, RosskampE, SchlüterC, SeifertB, Ullrich D. DEHP metabolites in urine of children and DEHP in house dust. Int J Hyg Environ Health. 207(5): 409-417. 2004 